\title{
LOS ARABINOXILANOS FERULADOS DE CEREALES. UNA REVISIÓN DE SUS CARACTERÍSTICAS FISICOQUÍMICAS Y CAPACIDAD GELIFICANTE
}

\section{FERULATED ARABINOXYLANS FROM CEREALS. A REVIEW OF THEIR PHYSICO-CHEMICAL CHARACTERISTICS AND GELLING CAPABILITY}

\author{
Adriana Morales-Ortega ${ }^{1}$, Guillermo Niño-Medina ${ }^{2}$, Elizabeth Carvajal-Millán ${ }^{1 \star}$, Alfonso Gardea-Béjar ${ }^{3}$, \\ Patricia Torres-Chávez ${ }^{4}$, Yolanda López-Franco ${ }^{1}$, Agustín Rascón-Chu ${ }^{1}$ y Jaime Lizardi-Mendoza ${ }^{1}$
}

\begin{abstract}
${ }^{1}$ Laboratorio de Biopolímeros, CTAOA, Centro de Investigación en Alimentación y Desarrollo (CIAD). km 0.6 Carr. a La Victoria. 83304, Hermosillo, Sonora. Tel: (662)2892400, Fax: (662)2800421. ${ }^{2}$ Facultad de Agronomía, Universidad Autónoma de Nuevo León. Francisco Villa s/n. 66050, Col. Ex-hacienda El Canadá, Escobedo, Nuevo León. ${ }^{3}$ CIAD Guaymas. Km 6.6 Carr. al Varadero Nacional, Col. Las Playitas. 85480, Guaymas, Sonora. ${ }^{4}$ Departamento de Investigación y Posgrado en Alimentos, Universidad de Sonora. Luis Encinas y Rosales s/n, Col. Centro. 83000, Hermosillo, Sonora.
\end{abstract}

*Autor para correspondencia (ecarvajal@ciad.mx)

\section{RESUMEN}

Los arabinoxilanos ferulados son los principales polisacáridos no amiláceos de los granos de cereales, que se localizan en las paredes celulares del endospermo, en la capa aleurona y en el pericarpio de los mismos. Estos polisacáridos se han reportado en los cereales más importantes, como trigo (Triticum aestivum $\mathrm{L}$.), centeno (Secale cereale L. M. Bieb.), cebada (Hordeum vulgare L.), avena (A. Sativa), arroz (Oryza sativa L.), sorgo (Sorghum vulgare), maíz (Zea mays L.) y mijo (Panicum miliaceum L.). Recientemente se han realizado esfuerzos enfocados a la extracción de arabinoxilanos ferulados a partir de subproductos de la industria procesadora de cereales, como de los pericarpios de maíz y de trigo, así como del "nejayote" que es el agua residual de la nixtamalización del maíz. Los arabinoxilanos ferulados forman soluciones viscosas y pueden formar geles bajo la acción de ciertos agentes oxidantes. Además, presentan características físicoquímicas determinantes para su capacidad gelificante. Los geles de arabinoxilanos han despertado un gran interés debido a que presentan características interesantes como: olor y sabor neutro; estabilidad al pH, a los cambios de temperatura y de concentración de electrolitos; así como una estructura macroporosa. Estas características les confieren aplicación potencial como matrices para la liberación controlada de biomoléculas en aplicaciones alimentarias y no alimentarias, lo cual podría dar valor agregado a los cereales o subproductos de cereales de los cuales pueden ser extraídos estos polisacáridos. Esta revisión incluye los reportes más recientes sobre las características fisicoquímicas y la capacidad gelificante de arabinoxilanos ferulados, tema sobre el cual ha resurgido el interés en los últimos diez años. La generación de nuevo conocimiento sobre este polisacárido y sus geles podría sentar las bases para su aplicación en distintas áreas como la agroalimentaria, la biomédica y la cosmética, entre otras.

Palabras clave: Arabinoxilanos, ácido ferúlico, gelificación, lacasa, dímeros, trímeros.

\section{SUMMARY}

FerulFerulated arabinoxylans are the primary non-starch polysaccharides in cereal grains. They are located in thecell walls of endosperm, aleurone layer and pericarp. There are reports of their presence in major cereal grains including wheat (Triticum aestivum L.), rye (Secale cereale L. M. Bieb.), barley (Hordeum vulgare L.), oat (A. Sativa), rice (Oryza sativa L.), sorghum (Sorghum vulgare), maize
(Zea mays L.) and millet (Panicum miliaceum L.). Recent research has focused on arabinoxylan extraction from low-value cereal byproducts in the food industry, such as bran cereal and 'nejayote' (maize nixtamalization wastewater). Ferulated arabinoxylans form highly viscous solutions, and they can form gels in the presence of certain oxidizing agents. Gelling capability of cereal arabinoxylans is determined by inherent physicochemical properties. Arabinoxylan gels are receiving increasing attention due to characteristics such as neutral odor and taste, stability to $\mathrm{pH}$ and electrolyte concentration changes, and macro porous structure. They can potentially be used as controlled-release matrices in food and non-food applications like biomedicine and cosmetics. Thus, cereal grains and by-products become more valuable. This review includes some of the most recent findings on the physicochemical properties and gelling capability of ferulated arabinoxylans from cereals.

Index words: Arabinoxylans, ferulic acid, oxidative coupling, laccase, dehydrodimers, dehydrotrimers.

\section{INTRODUCCIÓN}

Los arabinoxilanos (AX) son polisacáridos neutros no amiláceos de los granos de cereales. Algunos residuos de arabinosa se encuentran esterificados con el ácido ferúlico (ácido 3-metoxi-4-hidroxicinámico) en posición $(O)$-5, por lo que son llamados arabinoxilanos ferulados (Smith y Hartley, 1983). Varios subproductos del procesamiento de cereales se han investigado como posibles fuentes de arabinoxilanos ferulados con capacidad gelificante; tal es el caso de los pericarpios de maíz (Zea mays L.) y de trigo (Triticum aestivum L.), así como del "nejayote" (líquido residual de la nixtamalización del maíz). Existen algunos reportes sobre las características físico-químicas (peso molecular, viscosidad intrínseca, grado de sustitución) y funcionales (capacidad gelificante, propiedades texturizantes y emulsificantes) de arabinoxilanos ferulados extraídos del endospermo y el pericarpio de diversos cereales: trigo, centeno (Secale cereale L. M. Bieb.), cebada (Hordeum vulgare L.), maíz y arroz (Oryza sativa L.), entre otros (Rattan et 
al., 1994; Izydorczyk y Biliaderis, 1995; Vansteenkiste et al., 2004; Carvajal-Millan et al., 2005a, 2007).

Los arabinoxilanos ferulados son capaces de formar geles covalentes resistentes a cambios de $\mathrm{pH}$, de temperatura y de fuerza iónica. Estos geles no son degradados durante su paso por el sistema gastrointestinal. En esta revisión se describen los avances recientes de las características físicoquímicas de los arabinoxilanos ferulados en cereales y la relación con su capacidad gelificante, ya que esta propiedad funcional aumenta el potencial de aplicación de este polisacárido en las industrias alimentaria, biomédica y cosmética, entre otras.

\section{ESTRUCTURA QUÍMICA, LOCALIZACIÓN Y EXTRACCIÓN}

Los arabinoxilanos son un grupo heterogéneo de polisacáridos en el cual varían los patrones de sustitución y el grado de polimerización. Están formados por una cadena lineal de xilosas unidas por enlaces glicosídicos $\beta-(1 \rightarrow 4)$, a la cual se unen residuos de arabinosas mediante enlaces glicosídicos $\alpha-(1 \rightarrow 3)$ o $\alpha-(1 \rightarrow 2)$, o ambos (Figura 1$)$. La xilosa puede presentar dos grados de sustitución, de acuerdo con el número de residuos de arabinosa (monosustituído o disustituído). Además de las unidades de arabinosa es común encontrar otros sustituyentes minoritarios como son el ácido glucurónico y la galactosa (Izydorczyc y Biliaderis, 1995; Vinks y Delcour, 1996).

Los arabinoxilanos ferulados tienen la particularidad de presentar ácido ferúlico en su estructura, unido en la posición $O-5$ de arabinosa por medio de un enlace éster (Ishii, 1997; Saulnier y Thibault, 1999) (Figura 1). El término "grado de sustitución" utilizado en la descripción de los arabinoxilanos, se refiere al número de unidades de arabinosa unido a la cadena principal de xilosas, y también se descri- be como la relación arabinosa-xilosa (A/X). Tanto el grado de sustitución como la distribución de las cadenas laterales son factores importantes en las características fisicoquímicas de los arabinoxilanos (Grootaert et al., 2007).

En función de su solubilidad los arabinoxilanos se clasifican en: extraíbles en agua (WEAX, water extractable arabinoxylan) y no extraíbles en agua (WUAX, water unextractable arabinoxylan). Los WEAX son extraíbles a temperatura ambiente, mientras que los WUAX requieren de un tratamiento alcalino para su extracción. Además de su solubilidad, las principales diferencias entre los WEAX y los WUAX son que estos últimos son de un peso molecular más alto y poseen mayor diversidad en las cadenas laterales, ya que además de arabinosa contienen ácido glucurónico, galactosa y grupos acetilo (Saulnier et al., 2007).

En los cereales los WEAX se encuentran principalmente en el endospermo, y los WUAX se ubican tanto en la capa aleurona como en el pericarpio. La cantidad de arabinoxilanos en un tejido vegetal puede variar dependiendo del género, así como por los factores ambientales y los estadios de desarrollo (estado de madurez). En general, el contenido de WEAX es menor ( 0.5 a $3.0 \%, \mathrm{p} / \mathrm{p})$ en el endospermo de los cereales (trigo, centeno, cebada) que el de WUAX (20 a $30 \%, \mathrm{p} / \mathrm{p}$ ) en el pericarpio de los mismos (Izydorczyc y Biliaderis, 2007).

La mayoría de estudios sobre los arabinoxilanos de cereales han sido desarrollados por grupos de investigación europeos que han utilizado como fuente principal a variedades de trigo, cebada y centeno (Figueroa-Espinoza y Rouau, 1998; Schooneveld-Bergmans et al., 1999; Vansteenkiste et al., 2004; Carvajal-Millan et al., 2003, 2005a). Otras investigaciones se han enfocado a los subproductos que se generan por el procesamiento de cereales (Carvajal-Millán et al., 2007; Niño-Medina et al., 2009).

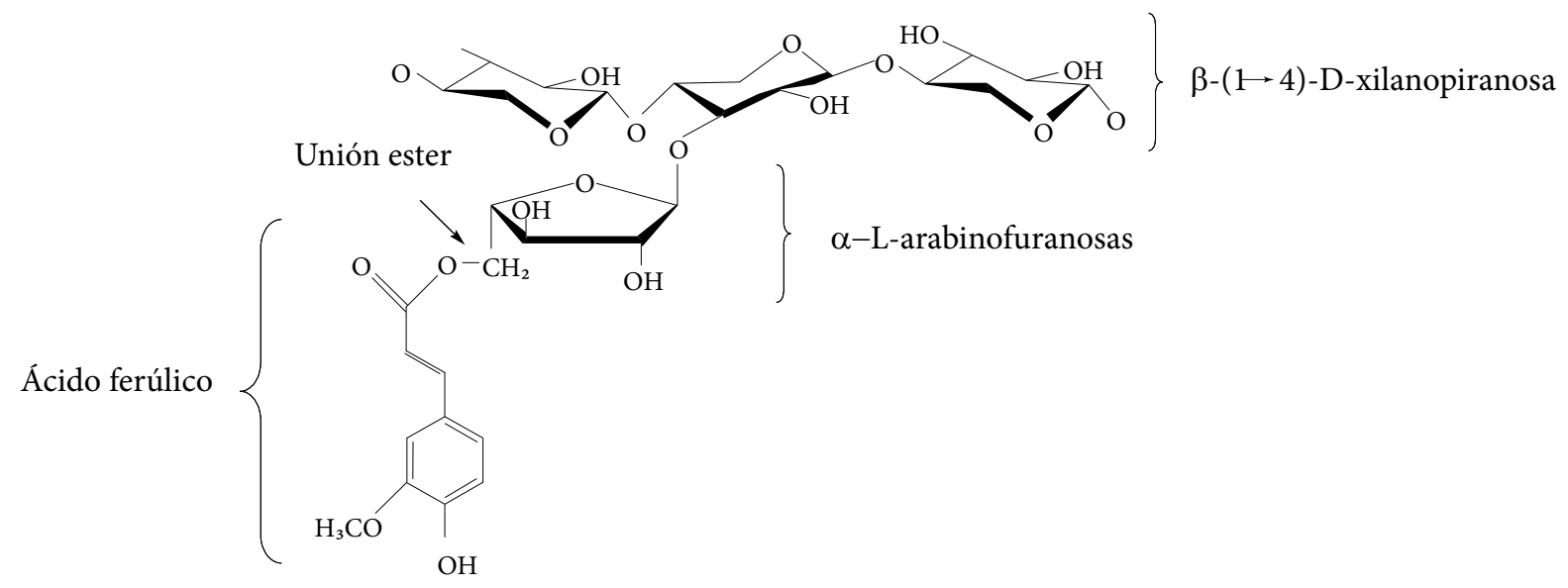

Figura 1. Estructura química de una fracción de arabinoxilanos ferulados identificados en cereales. 
En México, la industria productora de harina de maíz nixtamalizado, tales como Maseca y Minsa, genera anualmente grandes cantidades de pericarpio de maíz (20 000 a $46000 \mathrm{t}$ por empresa) y "nejayote" ( 3 a 6.9 millones de litros por empresa). Así mismo, la industria productora de harina de trigo, tales como Molino La Fama y Molino El Globo, obtiene pericarpio de trigo (15 $750 \mathrm{t}$ por empresa) como subproducto (Niño-Medina et al., 2010b). Estos subproductos contienen principalmente arabinoxilanos ferulados, por lo que la extracción de este polisacárido permitiría dar un uso alternativo a estos subproductos, ya que este polisacárido podría ser utilizado como texturizante, emulsificante o agente de encapsulación, entre otros usos.

La obtención de arabinoxilanos se lleva a cabo por extracción acuosa o extracción alcalina, ya sea usando el grano del cereal completo o tejidos específicos del mismo. La extracción acuosa es utilizada para obtener los WEAX principalmente a partir de harina de trigo, con un rendimiento de extracción entre 0.5 y 6 \% (p WEAX/p harina de trigo).

En la pared celular de pericarpio este polisacárido forma entrecruzamientos covalentes y físicos con otros constituyentes de la pared celular, lo que da como resultado una estructura insoluble en agua. En este caso se utiliza la extracción alcalina $\left(\mathrm{NaOH} 0.5 \mathrm{M}, \mathrm{Ca}(\mathrm{OH})_{2} 1.0 \mathrm{M}\right)$, con la cual los WUAX son separados de otros componentes de la pared celular para luego ser hidrolizados y transformados en cadenas más cortas y lograr su solubilidad en agua. Después de la extracción, los procedimientos de purificación normalmente incluyen el uso de proteasas y amilasas para la eliminación de proteína y almidón, respectivamente (Autio, 2006; Izydorczyk y Biliaderis, 2007).

\section{CARACTERÍSTICAS FISICOQUÍMICAS}

Peso molecular. Una de las variables más importantes para caracterizar una macromolécula es su peso molecular. El peso molecular de los arabinoxilanos depende de su origen y del método utilizado para su determinación. Por ejemplo, en el caso de WEAX de trigo los primeros estudios mediante técnicas de sedimentación por centrifugación reportaron valores de peso molecular muy bajos (65 $\mathrm{kDa}$ ), mientras que con cromatografía de filtración en gel se encontraron pesos moleculares muy elevados $(\geq 800$ kDa) (Izydorczyk y Biliaderis, 2007). Por su parte, Biliaderis (1995) reportó un peso molecular en el intervalo de 134 a $201 \mathrm{kDa}$ en WEAX extraídos de distintas variedades de trigo canadiense, mientras que Dervilly et al. (2000) y Dervilly-Pinel et al. (2001a; b) reportaron que el peso molecular promedio de los WEAX de trigo se encuentra entre $200 \mathrm{y}$ $300 \mathrm{kDa}$. Para WUAX de pericarpio de maíz, Lapierre et al. (2001) encontraron una variación de pesos moleculares de 124 hasta $270 \mathrm{kDa}$ con distintos métodos de extracción.
Según Shyama y Muralikrishna (2007), el peso molecular de WEAX de arroz es de $231 \mathrm{kDa}$, que disminuye a $75 \mathrm{kDa}$ después del proceso de malteado (hidrólisis enzimática del almidón del cereal).

Viscosidad intrínseca. La viscosidad intrínseca ([h]) es un indicador del volumen hidrodinámico ocupado por un polímero, que depende del peso molecular y de la conformación del polímero en el solvente. Para los WEAX de trigo la viscosidad intrínseca se encuentra entre 200 y 600 $\mathrm{mL} / \mathrm{g}$, con un promedio de $400 \mathrm{~mL} / \mathrm{g}$ (Izydorczyc et al., 1991; Rattan et al., 1994; Saulnier et al., 1995). CarvajalMillán et al. (2007) reportaron una viscosidad intrínseca de $208 \mathrm{~mL} / \mathrm{g}$ para WUAX obtenidos de pericarpio de maíz. La menor viscosidad intrínseca de los WUAX se debe a que para poder ser extraídos en agua, deben ser previamente hidrolizados para reducir el tamaño de la molécula. El valor de $[h]$ define el potencial de aplicación de los WEAX o los WUAX. Un valor alto de $[h]$ favorece la capacidad gelificante del polisacárido, mientras que un valor bajo mejora sus propiedades probióticas.

Solubilidad. La solubilidad de los arabinoxilanos depende en gran medida del grado de sustitución que presenta la xilosa con unidades de arabinosa. El contenido de sustituyentes de arabinosa, así como su distribución a lo largo de la cadena de xilosa, afecta el potencial de las cadenas de arabinoxilanos para interactuar entre sí o con otros polisacáridos.

Para determinar el efecto de la sustitución de arabinosas sobre la solubilidad de los arabinoxilanos, Andrewartha et al. (1979) prepararon una serie de arabinoxilanos solubles en agua purificados de harina de trigo, en los que removieron parcialmente uniones laterales de arabinosa mediante el uso de $\alpha$-L-arabinofuranosidasa; estos autores encontraron que conforme disminuye la proporción de $\mathrm{A} / \mathrm{X}$, ocurre una disminución significativa de la solubilidad de los arabinoxilanos, y como valor crítico reportaron una proporción A/X de 0.43 a partir de la cual la solubilidad disminuye en forma abrupta.

Para los cereales (principalmente trigo, centeno, cebada, arroz, maíz) el valor A/X es generalmente más alto en los WUAX (0.6 a 0.8) que en los WEAX (0.5 a 0.7) (Izydorczyk y Biliaderis, 2007). Algunas investigaciones señalan un mayor valor de $\mathrm{A} / \mathrm{X}$ en cereales cultivados bajo condiciones de estrés hídrico. Sternemalm et al. (2008) estudiaron el efecto de la sustitución de arabinosa en la solubilidad de los arabinoxilanos mediante la utilización de soluciones a $1 \%$ de arabinoxilanos y una relación $\mathrm{A} / \mathrm{X}$ inicial de 0.52 . Estos autores observaron que conforme decrecía la relación $\mathrm{A} / \mathrm{X}$ también disminuía la solubilidad de los arabinoxilanos, y su agregación parcial se iniciaba en el rango A/X entre 0.31 
y 0.23 , y precipitaba totalmente a un valor A/X aproximado a 0.1. Este comportamiento se debe a que los residuos de arabinosa favorecen la interacción del polisacárido con el agua. Una vez removidas las sustituciones de arabinosa, las cadenas de xilosas pueden formar interacciones estables entre las cadenas, que confieren una conformación muy rígida a los arabinoxilanos y producen agregados insolubles.

\section{CAPACIDAD GELIFICANTE}

Mecanismo de gelificación. Las soluciones acuosas de arabinoxilanos ferulados tienen la característica de formar geles covalentes en presencia de agentes generadores de radicales libres. Estos agentes pueden ser enzimáticos vía lacasa $/ \mathrm{O}_{2} \mathrm{o}$ peroxidasa/ $\mathrm{H}_{2} \mathrm{O}_{2}$ (Figueroa-Espinoza y Rouau, 1998) o químicos (persulfato de amonio y cloruro férrico) (Niño-Medina et al., 2010a). Los enlaces covalentes, que unen las cadenas de arabinoxilanos a través del ácido ferúlico, son los responsables de esta característica especial en el polisacárido (Figura 2). Las enzimas comúnmente utilizadas para la formación de geles de arabinoxilanos son lacasa/ $\mathrm{O}_{2}$ y peroxidasa $/ \mathrm{H}_{2} \mathrm{O}_{2}$, y aunque difieren los mecanismos de acción de estas dos enzimas sus productos finales son los mismos (Autio, 2006).

La catálisis enzimática de la lacasa (E.C.1.10.3.2) está basada en un mecanismo oxidativo en el que el oxígeno es el aceptor final de electrones. La lacasa cataliza la oxidación del ácido ferúlico. reacción que se encuentra acoplada a la reducción de oxígeno molecular con cuatro electrones para formar agua. Se necesita un total de cuatro moléculas de ácido ferúlico para llevar a cabo esta reacción a término (Selinheimo et al., 2007; Calcaterra et al., 2008).

La peroxidasa cataliza la oxidación del ácido ferúlico, reacción que usa al peróxido de hidrógeno como agente oxidante. Esta es una reacción cíclica de tres pasos, en la cual la enzima peroxidasa $\left(\mathrm{Fe}^{3+}\right)$ primero es oxidada por el peróxido de hidrógeno. Los electrones son reemplazados en dos pasos secuenciales de transferencia de un electrón, en donde el ácido ferúlico reducido se convierte en ácido ferúlico oxidado. La reacción completa utiliza dos moléculas de ácido ferúlico (Halliwell, 1978; Selinheimo et al., 2007).

Es importante remarcar que tanto la lacasa como la peroxidasa- $\mathrm{H}_{2} \mathrm{O}_{2}$ sólo inducen la oxidación del ácido ferúlico, mientras que la formación de dímeros, trímeros y oligómeros de ácido ferúlico ocurre completamente al azar mediante un mecanismo de radicales. Debido a que los enlaces que unen las cadenas de arabinoxilanos por medio del ácido ferúlico son de tipo covalente, estos geles se encuentran clasificados como geles químicos, lo que les confiere la particularidad de ser irreversibles (Papon et al., 2006).
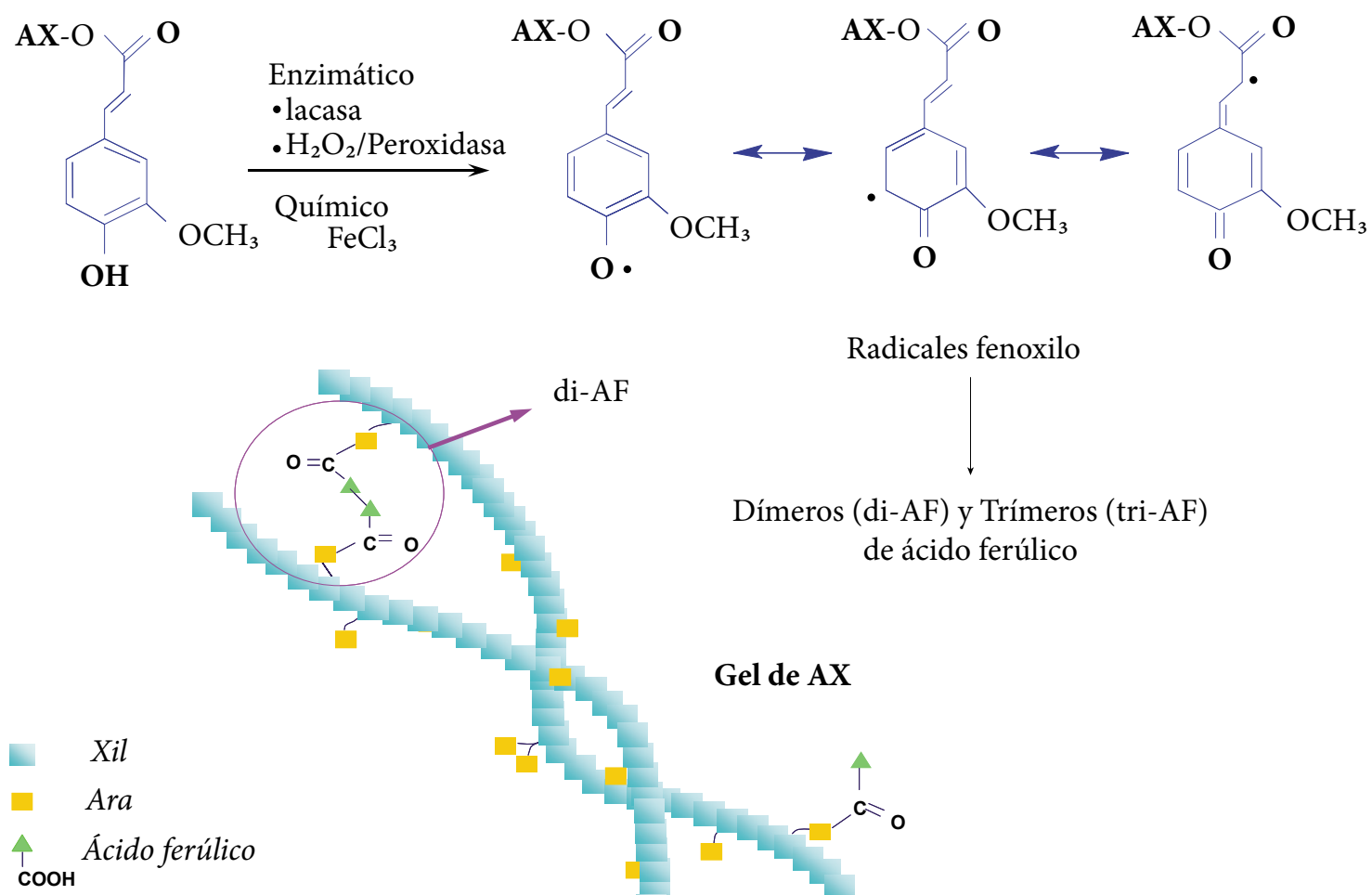

Radicales fenoxilo

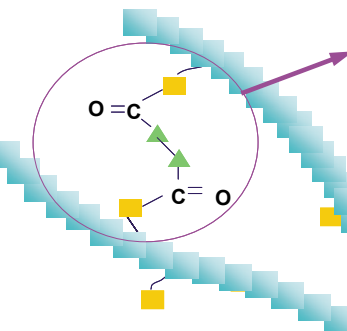

di-AF

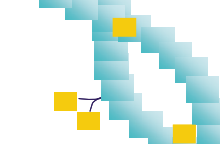

Gel de AX

Dímeros (di-AF) y Trímeros (tri-AF)

de ácido ferúlico

Figura 2. Representación esquemática de la gelificación de los arabinoxilanos ferulados. 
Características físico-químicas de los geles de arabinoxilanos. Estos geles presentan características muy particulares: son neutros, incoloros e inodoros, y poco afectados por cambios de temperatura, de fuerza iónica o de $\mathrm{pH}$, debido a la presencia de enlaces covalentes (Izydorczyk y Biliaderis, 1995). Carvajal-Millan et al. (2006) evaluaron las propiedades estructurales y reológicas de geles de arabinoxilanos preparados mediante la acción de la enzima lacasa, y concluyeron que estas características pueden ser controladas mediante la modificación química del contenido de ácido ferúlico del arabinoxilano.

La deferulación parcial de los arabinoxilanos reduce el contenido de enlaces covalentes en el gel, lo cual se refleja en la disminución del módulo elástico y el incremento en el tamaño de poro del gel. Una disminución de $40 \%$ del contenido inicial de ácido ferúlico reduce hasta $89 \%$ la elasticidad del gel formado (Carvajal-Millan et al., 2005a). A concentraciones de arabinoxilanos mayores de $1 \%(\mathrm{p} / \mathrm{v})$, no hay incremento en el contenido de dímeros y trímeros de ácido ferúlico, aun cuando el módulo elástico continúa incrementando y el tamaño de poro de la red disminuye, lo cual denota la acción de otras estructuras ferúlicas que crean los enlaces covalentes o interacciones físicas en la estructura del gel.

Hasta el momento se han identificado cinco formas isoméricas de di-AF en geles de arabinoxilanos (5-5, 8-5' benzo, 8-O-4', 8-5' y 8-8') (Figura 3). Las formas 8-5' y 8-O-4' son generalmente más abundantes en geles de arabinoxilanos de trigo (Figueroa-Espinoza y Rouau, 1998; Schooneveld-Bergmans et al., 1999; Vansteenkiste et al., 2004; Carvajal-Millan et al., 2005a, b, 2006). En cambio, en geles de arabinoxilanos de maíz el di-AF 5-5' se ha reportado como mayoritario (Carvajal-Millán et al., 2007; Niño-Medina et al., 2009; Berlanga-Reyes et al., 2009a). Hasta el momento se ha reportado la formación de un solo trímero de ácido ferúlico (tri-AF) (ácido 4-O-8', 5'-5”-dehidrotriferúlico) en geles de arabinoxilanos de trigo y de maíz (Carvajal-Millan et al., 2005a, 2007) inducidos por una lacasa.

La forma isomérica del di-AF puede tener un efecto en la elasticidad del gel de arabinoxilanos, ya que no todos son efectivos en generar entrecruzamientos entre cadenas distintas del polisacárido. Según Hatfield et al. (1999), el isómero 5-5' es el único que puede formarse tanto entre cadenas distintas (intercadena) como en una misma cadena de polisacárido (intracadena). Este último tipo de unión no contribuye a la elasticidad del gel.

En la formación del tri-AF en los geles de arabinoxilanos, la proporción que determina la elasticidad del gel aún no está bien definida. En general, más de $90 \%$ del contenido inicial de ácido ferúlico presente en los arabinoxilanos es oxidado al formarse el gel y sólo $30 \%$ se recupera en formas de di y tri-AF conocidas hasta el momento. Algunos autores señalan la formación de estructuras de di y tri-AF distintas a las que han sido identificadas, o bien de oligómeros superiores de ácido ferúlico (tetrámeros, pentámeros) (Vansteenkiste et al., 2004; Carvajal-Millan et al., 2005a, 2006, 2007).

Bunzel et al. $(2005 ; 2006)$ aislaron e identificaron dos nuevos trímeros de ácido ferúlico (ácido 8-5(no-cíclico)/55-dehidrotriferúlico y ácido 5-5-dehidrotriferúlico) y, por primera vez en pericarpio de maíz, dos tetrámeros de ácido ferúlico (ácido 4-O-8/5-5/8-O-4-dehidrotetraferúlico y ácido 4-O-8/5-5/8-5(no-cíclico)-dehidrotetraferúlico). Estas nuevas estructuras no han sido aún estudiadas en geles de arabinoxilanos, pero dado que en la naturaleza su función es unir estos polisacáridos para dar soporte estructural a la pared celular, es muy probable que se formen durante el proceso de gelificación del polisacárido.

Además de la participación de las estructuras di-AF y tri-AF anteriormente mencionados, algunos autores han señalado la contribución de interacciones físicas entre las regiones lineales de las cadenas de arabinoxilanos en el establecimiento de la estructura tridimensional del gel (Vansteenkiste et al., 2004; Carvajal-Millan et al., 2005b).

La presencia de cadenas laterales de xilosa se ha detectado en arabinoxilanos de trigos europeos (Adams et al., 2003), las cuales pueden afectar el acercamiento de las cadenas de arabinoxilanos y el establecimiento de la estructura del gel. La estructura del gel de arabinoxilanos estaría entonces determinada tanto por los enlaces covalentes de ácido ferúlico (di, tri-AF), como por las interacciones físicas entre las regiones lineales de xilosa, lo cual a su vez puede afectar los parámetros estructurales y las propiedades funcionales del gel formado.

La estructura macroporosa de los geles de arabinoxilanos con un tamaño de poro entre 40 y $400 \mathrm{~nm}$ (Cuadro 1) confiere a los arabinoxilanos un alto potencial de aplicación en la encapsulación y transporte de biomoléculas como proteínas o péptidos terapéuticos, los cuales al suministrarse por vía oral son susceptibles a degradación enzimática durante su paso por el tracto gastrointestinal (Vansteenkiste et al., 2004; Carvajal-Millan et al., 2005b).

Las biomoléculas modelo, hasta el momento estudiadas en geles de arabinoxilanos, son la seroalbúmina de bovino (BSA), la ovoalbúmina y la insulina, entre otras (CarvajalMillan et al., 2005b; Berlanga-Reyes et al., 2009b). Estos estudios se han realizado en geles a 1 y $2 \%$ de arabinoxilanos que contienen de 0.1 a $10 \%$ de la proteína modelo encapsulada. Este amplio rango de carga de proteína en el 
<smiles>COc1cc(/C=C/C(=O)O)cc(/C(=C/c2ccc(O)c(CO)c2)C(=O)O)c1O</smiles>

(a)<smiles>COC1=CC(CCC(=O)O)C[Ge](C(=O)O)OC1=Cc1ccc(O)c(OC)c1</smiles>

(b)<smiles>COc1cc(/C=C/C(=O)O)cc(-c2cc(/C=C(/Oc3ccc(/C=C/C(=O)O)cc3C)C(=O)O)cc(C)c2O)c1O</smiles>

Figura 3. Estructura química de los dímeros (a) y trímeros (b) del ácido ferúlico, identificados en geles de arabinoxilanos. Adaptado de Niño-Medina et al. (2009).

Cuadro 1. Parámetros estructurales de geles de arabinoxilanos inducidos por la enzima lacasa.

\begin{tabular}{|c|c|c|c|c|c|c|c|}
\hline $\begin{array}{l}\text { Enzima comercial } \\
(0.04 \mathrm{U} / \mathrm{mg} \mathrm{AX})\end{array}$ & Fuente & $\mathrm{AX}^{\dagger}(\% \mathrm{p} / \mathrm{v})$ & $\begin{array}{c}\mathrm{di}-\mathrm{AF}^{\dagger \dagger} \\
(\mu \mathrm{g} / \mathrm{mg})\end{array}$ & $\begin{array}{l}\text { tri-AFg } \\
(\mu \mathrm{g} / \mathrm{mg})\end{array}$ & $\xi 99(\mathrm{~nm})$ & $\begin{array}{c}\mathrm{Mc}^{\mathrm{x}} \times 10^{3} \\
(\mathrm{~g} / \mathrm{mol})\end{array}$ & $\begin{array}{c}\rho_{\mathrm{c}}^{\mathrm{xx}} \times 10^{6} \\
\left(\mathrm{~mol} / \mathrm{cm}^{3}\right)\end{array}$ \\
\hline T. versicolor & Maíz & 2.5 & 0.029 & 0.014 & 80 & 31 & 49 \\
\hline T. versicolor & Maíz & 3.5 & 0.030 & 0.020 & 48 & 20 & 75 \\
\hline P. cinnabarinus & Trigo & 2.0 & 0.029 & 0.013 & 305 & 145 & 12 \\
\hline
\end{tabular}


gel permitiría una amplia gama de aplicaciones potenciales para este sistema de transporte. Una de estas aplicaciones potenciales sería la liberación controlada de insulina dirigida al colon (Berlanga-Reyes et al., 2009b; Carvajal-Millan et al., 2013).

Anteriormente se había reportado una relación directa entre los parámetros estructurales del gel de arabinoxilanos (tamaño de poro, densidad de puntos de unión, distancia entre dos puntos de unión) y sus propiedades funcionales (capacidad de retención de agua al equilibrio y capacidad para liberar biomoléculas de manera controlada) (Carvajal-Millan et al., 2005b). Pero hasta el momento no ha sido posible cuantificar la contribución relativa de los enlaces covalentes y de las interacciones físicas en la formación del gel de arabinoxilanos.

\section{CONCLUSIONES}

Los arabinoxilanos ferulados son polisacáridos recuperados principalmente de granos de cereales, y más recientemente de subproductos de la industria procesadora de cereales. Una de las principales características de los arabinoxilanos ferulados es la capacidad de formar geles covalentes estables a cambios de $\mathrm{pH}$ y de temperatura. A estos geles se les atribuye un gran potencial de aplicación como matrices para la liberación controlada de biomoléculas en industrias alimentarias y no alimentarias.

Los arabinoxilanos ferulados continúan siendo investigados y se sigue generando nueva información sobre este tema. Sin embargo, se requiere más investigación para conocer más sobre la formación de estructuras de entrecruzamiento superiores de ácido ferúlico durante la gelificación enzimática de estos polisacáridos. Se requiere también demostrar la contribución real de estas nuevas estructuras en la formación del gel.

Además, es necesario realizar estudios complementarios acerca de otras propiedades funcionales de los arabinoxilanos ferulados de cereales, como las probióticas y antioxidantes. La generación de nuevo conocimiento sobre este polisacárido y sus geles podría sentar las bases para su aplicación como biomaterial en la industria agroalimentaria, biomédica o cosmética, entre otras, que le permitiría dar valor agregado a los cereales o subproductos de cereales de los cuales puede ser extraído.

\section{AGRADECIMIENTOS}

Al Fondo Institucional SEP-CONACYT, México (proyecto clave 134301 aprobado a E. Carvajal-Millán). A Alma C. Campa Mada, Alma Rosa Toledo Guillén y Karla G. Martínez-Robinson del CIAD, por su participación en este proyecto.

\section{BIBLIOGRAFÍA}

Adams L E, P A Kroon, G Williamson, V J Morris (2003) Characterization of heterogeneous arabinoxylans by direct imaging of individual molecules by atomic force microscopy. Carbohydrate Res. 338:771-780.

Andrewartha K A, D R Phillips, B A Stone (1979) Solution properties of wheat-flour arabinoxylans and enzimatically modified arabinaoxylans. Carbohydrate Res. 77:191-204.

Autio K (2006) Functional aspects of cereal cell-wall polysaccharides. In: Carbohydrates in Food. A-C Eliasson (ed). CRC-Taylor \& Francis. Boca Raton, FLA. pp:167-207.

Berlanga-Reyes C M, E Carvajal-Millán, G C Juvera, A Rascón-Chu, J A Márquez- Escalante, A L Martínez-López (2009a) Laccase induced maize bran arabinoxylan gels: Structural and rheological properties. Food Sci. Biotech. 18:1027-1029.

Berlanga-Reyes C M, E Carvajal-Millán, J Lizardi-Mendoza, A RascónChu, J A Márquez-Escalante, A L Martínez-López (2009b) Maize arabinoxylan gels as protein delivery matrices. Molecules 14:1475-1482.

Biliaderis C G (1995) Effect of arabinoxylan on bread-making quality of wheat flours. Food Chem. 53:165-171.

Bunzel M, J Ralph, C Funk, H Steinhart (2005) Structural elucidation of new ferulic acid-containing phenolic dimers and trimers isolated from maize bran. Tetrahedron Lett. 46:5845-5850.

Bunzel M, J Ralph, P Bruning, H Steinhart (2006) Structural identification of dehydrotriferulic and dehydrotetraferulic acids isolated from insoluble maize bran fiber. J. Agric. Food Chem. 54:64096418.

Calcaterra A, C Galli, P Gentili (2008) Phenolic compounds as likely natural mediators of laccase: a mechanistic assessment. J. Mol. Catalysis B: Enzymatic 51:118-20.

Carvajal-Millán E, C Berlanga-Reyes, A Rascón-Chu, A L MartínezLópez, J A Márquez-Escalante, A C Campa-Mada, K G Martínez-Robinson (2013) In vitro evaluation of arabinoxylan gels as an oral delivery system for insulin. In: Material Research Society Symposium Proceedings. S E Rodil, A Almaguer-Flores, K Anselme y J Castro (eds). Cancún MEX. 12-17. Ago. Vol. 1487. DOI: $10.1557 / o p l .2012 .1529$.

Carvajal-Millán E, A Rascón-Chu, J A Márquez-Escalante, V Micard, N Ponce de León, A Gardea (2007) Maize bran gum: extraction, characterization and functional properties. Carbohydr. Polym. 69:280-285.

Carvajal-Millan E, S Guilbert, J L Doublier, V Micard (2006) Arabinoxylan/protein gels: Structural, rheological and controlled release properties. Food Hydrocolloids 20:53-61.

Carvajal-Millan E, V Landillon, M H Morel, X Rouau, J L Doublier, V Micard (2005a) Arabinoxylan gels: impact of feruloylation degree on their structure and properties. Biomacromolecules 6:309-317.

Carvajal-Millan E, S Guilbert, M H Morel, V Micard (2005b) Impact of the structure of arabinoxylan gels on their rheological and protein transport properties. Carbohydr. Polym. 60:431-438.

Carvajal-Millan E, A Surget, X Rouau, S Guilbert, V Micard (2003) Arabinoxylan networks as affected by ovalbumin content. Macromolecular Symposia 200:129-136.

Dervilly-Pinel G, J F Thibault, L Saulnier (2001a) Experimental evidence for a semi-flexible conformation for arabinoxylans. Carbohydr. Res. 330:365-72.

Dervilly G, L Saulnier, P Roger, J F Thibault (2000) Isolation of homogeneous fractions of wheat water-soluble arabinoxylan. Influence of structure on their macromolecular characteristics. J. Agric. Food Chem. 48:270-278.

Figueroa-Espinoza M C, X Rouau (1998) Oxidative crosslinking of pentosans by a fungal laccase and horseradish peroxidase: mechanism of linkage between feruloylated arabinoxylans. Cereal Chem. 75:259-265.

Grootaert C, J A Delcour, C M Courtin, W F Broekaert, W Verstraete, T Van de Wiele (2007) Microbial metabolism and prebiotic potency of arabinoxylan oligosaccharides in the human intestine. Trends Food Sci. Technol. 18:64-71.

Halliwell B (1978) Lignin synthesis: the generation of hydrogen peroxide and superoxide by horseradish peroxidase and its stimulation 
by manganese (II) and phenols. Planta 140:81-88.

Hatfield R D, J Ralph, J H Grabber (1999) Cell wall cross-linking by ferulates and diferulates in grasses. J. Sci. Food Agric. 79:403-407.

Ishii T (1997) Structure and functions of feruloylated polysaccharides. Plant Sci. 127:111-127.

Izydorczyk M S, C G Biliaderis (2007) Arabinoxylans: Technologically and nutritionally functional plant polysaccharides. In: Functional Food Carbohydrates. C G Biliaderis, M S Izydorczyk (eds). CRC Press, Boca Raton, FL, USA. pp:249-290.

Izydorczyk M S, C G Biliaderis (1995) Cereal arabinoxylan: advances in structure ad physicochemical properties. Carbohydr. Polym. 28:33-48.

Izydorczyk M S, C G Biliaderis, W Bushuk (1991) Physical-properties of water-soluble pentosans from different wheat- varieties. Cereal Chem. 68:145-150.

Lapierre C, B Pollet, M C Ralet, L Saulnier (2001) The phenolic fraction of maize bran: evidence for lignin-heteroxylan association. Phytochemistry 57:765-772.

Niño-Medina G, E Carvajal-Millan, J Lizardi, A Rascón-Chu, A Gardea (2010b) Feruloylated arabinoxylans recovered from low - value maize by - products. In: Handbook of Carbohydrate Polymers: Development, Properties and Applications. R Ito, Y Matsuo (eds). Nova Science Publishers, Inc. New York. pp:711-725.

Niño-Medina G, E Carvajal-Millan, J Lizardi, A Rascon-Chu, J A Marquez-Escalante, A Gardea, V Guerrero, E Salas-Muñoz (2010a) Feruloylated arabinoxylans and arabinoxylan gels: structure, sources and applications. Phytochemistry 9:111-120.

Niño-Medina G, E Carvajal-Millán, J Lizardi, A Rascon-Chu, J A Marquez-Escalante, A Gardea, A L Martinez-Lopez, V Guerrero (2009) Maize processing waste water arabinoxylans: gelling capability and cross-linking content. Food Chemistry 115:12861290.

Papon P, J Leblond, P Meijer (2006) Gelation and transitions in biopolymers. In: The Physics in Phase Transition. Concepts and Applications. 2nd ed. Springer-Verlag. New York. pp:189-213.
Rattan O, M S Izydorczyk, C G Biliaderis (1994) Structure and rheological behaviour of arabinoxylans from Canadian bread wheat flours. Lebensm.-Wiss. Technol. 27:550-555.

Saulnier L, P E Sado, G Branlard, G Charmet, F Guilon (2007) Wheat arabinoxylan: exploiting variation in amount and composition to develop enhanced varieties. J. Cereal Chem. 46:261-281.

Saulnier L, J F Thibault (1999) Ferulic acid and diferulic acids as components of sugar-beet pectins and maize bran heteroxylans. J. Sci. Food Agric. 79:396-402.

Saulnier L, N Peneau, J F Thibault (1995) Variability in grain extract viscosity and water-soluble arabinoxylan content in wheat. J. Cereal Sci. 22:259-264.

Schooneveld-Bergmans M E F, M J W Dignum, J H Grabber, G Beldman, A G J Voragen (1999) Studies on oxidative cross-linking of feruloylated arabinoxylans from wheat flour and wheat bran. Carbohydr. Polym. 38:309-317.

Selinheimo E, K Autio, K Kruus, J Buchert (2007) Elucidating the mechanism of laccase and tyrosinase in wheat bread making. J. Agric. Food Chem.55:6357-6365.

Shyama P R R, G Muralikrishna (2007) Structural characteristics of water-soluble feruloyl arabinoxylans from rice (Oryza sativa) and ragi (Finger millet, Eleusine coracana): variations upon malting. Food Chem. 104:1160-1170.

Smith M M, R D Hartley (1983) Occurrence and nature of ferulic acid substitution of cell-wall polysaccharides in graminaceous plants. Carbohydr. Res. 118:65-80.

Sternemalm E, A Höije, P Gatenholm (2008) Effect of arabinose substitution on the material properties of arabinoxylan films. Carbohydr. Res. 343:753-757.

Vansteenkiste E, C Babot, X Rouau, V Micard (2004) Oxidative gelation of feruloylated rabinoxylan as affected by protein. Influence on protein enzymatic hydrolysis. Food Hydrocolloids 18:557-564.

Vinks C J A, J A Delcour (1996) Rye (Secale cereale L.) arabinoxylan. A critical review. J. Cereal Sci. 24:1-14. 